\title{
Indication of suitable transarterial chemoembolization and multikinase inhibitors for intermediate stage hepatocellular carcinoma
}

\author{
SHIGEO SHIMOSE $^{1}$, TAKUMI KAWAGUCHI ${ }^{1}$, HIDEKI IWAMOTO ${ }^{1,2}$, TAKASHI NIIZEKI ${ }^{1}$, \\ TOMOTAKE SHIRONO $^{1}$, MASATOSHI TANAKA ${ }^{3}$, HIRONORI KOGA ${ }^{1,2}$ and TAKUJI TORIMURA ${ }^{1,2}$ \\ ${ }^{1}$ Division of Gastroenterology, Department of Medicine; ${ }^{2}$ Division of Liver Cancer Research, Research Center for \\ Innovative Cancer Therapy, Kurume University School of Medicine, Kurume 830-0011; ${ }^{3}$ Department of \\ Gastroenterology and Hepatology, Yokokura Hospital, Miyama 839-0295, Japan
}

Received June 5, 2019; Accepted November 6, 2019

DOI: $10.3892 / 01.2020 .11399$

\begin{abstract}
Prognosis of patients with intermediate stage hepatocellular carcinoma (HCC) treated with transcatheter arterial chemoembolization (TACE) is unsatisfactory. The present study analyzed the indications for suitable TACE in patients with intermediate stage HCC. Additionally, it was investigated whether further TACE or switching to multi-kinase inhibitors (MKIs) was more beneficial for patients with HCC recurrence following initial TACE. The present retrospective study included 238 patients with intermediate stage HCC who were initially treated with TACE (median age, 74 years). A decision-tree analysis was employed to investigate the therapeutic effect profiles and overall survival (OS) rates. In the decision-tree analysis for OS, complete response (CR) by initial TACE was selected as the most important variable. In the decision-tree analysis for CR, $<3$ liver segments with nodule, simple nodular type and within the up-to-seven criteria were selected as the first, second and third variables associated with a high CR rate (35-64\%), respectively. In
\end{abstract}

Correspondence to: Dr Shigeo Shimose, Division of Gastroenterology, Department of Medicine, Kurume University School of Medicine, 67 Asahi-machi, Kurume 830-0011, Japan E-mail: shimose_shigeo@med.kurume-u.ac.jp

Abbreviations: HCC, hepatocellular carcinoma; TACE, transcatheter arterial chemoembolization; MKIs, multi-kinase inhibitors; BCLC, Barcelona Clinic Liver Cancer; OS, overall survival; PS, performance status; AFP, alpha-fetoprotein; DCP, des- $\gamma$-carboxy prothrombin; DC, drug-eluting; mRECIST, modified response evaluation criteria in solid tumors; AEs, adverse events; SAEs, serious adverse events; CTCAE, common terminology criteria for adverse events; CR, complete response; PR, partial response; $\mathrm{SD}$, stable disease; $\mathrm{PD}$, progressive disease; $\mathrm{MST}$, median survival time

Key words: hepatoma, prognosis, interventional radiology, tyrosine kinase inhibitor, exploratory data analysis patients with HCC recurrence having $\geq 3$ liver segments with nodule, out of the up-to-seven criteria, and Child-Pugh class A, the median survival time was significantly longer in those who were treated by switching to MKIs compared with further TACE (44.9 vs. 21.9 months; $\mathrm{P}=0.003$ ). In intermediate stage HCC, the indications for suitable TACE criteria may be ' $<3$ liver segments with nodule', 'simple nodular type', and 'within the up-to-seven criteria'. Additionally, in patients who were ineligible for TACE criteria, the switch to MKIs may improve the prognosis compared with further TACE in cases of HCC recurrence following first TACE.

\section{Introduction}

Hepatocellular carcinoma (HCC) is a common cause of cancer-related death worldwide (1). Only $30 \%$ of patients with HCC receive potentially curative therapies worldwide (2-5). Recently, there has been an increase in the number of patients with intermediate $\mathrm{HCC}$, mainly due to frequent recurrence/progression after treatment of $\mathrm{HCC}$ and an increase in the prevalence of non-viral HCC, including nonalcoholic steatohepatitis-related HCC (6-8). The majority of patients with intermediate or advanced-stage HCC generally undergo palliative treatments such as transcatheter arterial chemoembolization (TACE) (9-11) and systemic chemotherapy including multi-kinase inhibitors (MKIs) therapy (12-14).

TACE is a standard therapy for unresectable intermediate HCC, especially for patients with Barcelona Clinic Liver Cancer (BCLC) stage B (15). Several studies have shown that TACE significantly improves patient survival compared to the best supportive care and prolongs survival in patients with multiple HCC tumors and no macrovascular invasion $(11,16,17)$. However, further TACE could be associated with a high rate of treatment failure, worsening liver function, and poor prognosis in patients with HCC recurrence after TACE (18). Since tumor factors vary in the intermediate stage of HCC, it is important to identify the indications for suitable TACE in patients with HCC.

Recurrence/progression of $\mathrm{HCC}$ is frequently seen after initial TACE. For recurrent HCC, further TACE is a 
therapeutic option that can result in complete response (CR) even in patients with advanced HCC and portal vein tumor thrombosis (19). On the other hand, sorafenib, an MKI, is a standard first-line systemic treatment for advanced HCC $(20,21)$. Lenvatinib is a newly developed MKI that has been shown to be non-inferior to sorafenib in overall survival (OS) (22) and has been approved as a first-line systemic treatment for advanced HCC (22). Sorafenib is reported to improve OS and time to progression in patients with intermediate or advanced HCC that is refractory to TACE $(23,24)$. Moreover, the Transcatheter Arterial Chemoembolization Therapy in Combination with Sorafenib (TACTICS) trial showed that the combination of TACE with sorafenib significantly improved the time to progression compared to TACE alone in patients with HCC $(25,26)$. However, it remains unclear if further TACE or switching to MKIs is more beneficial for patients with HCC recurrence after TACE.

The aim of this study was to identify the indications for suitable TACE in patients with intermediate stage HCC. We also investigated whether further TACE or switching to MKIs was more beneficial for patients with HCC recurrence after initial TACE.

\section{Patients and methods}

Study design. This retrospective study was carried out in a single institution. The study protocol conformed to the ethical guidelines of the 1975 Declaration of Helsinki, as reflected by the prior approval of the ethical committee of Kurume University School of Medicine (approved no: 17205). An opt-out approach was used to obtain informed consent from the patients, and personal information was protected during data collection.

Patients. A total of 385 consecutive patients with HCC underwent TACE between 2009 and 2016 and were registered at the Kurume University School of Medicine. Patients meeting any of the exclusion criteria below were excluded from the analysis $(n=147)$. A total of 238 patients were included in this study. Among the included patients, 204 patients had been previously treated with radiofrequency ablation or hepatic rejection, and no patients had previously been treated with TACE.

Inclusion and exclusion criteria. The following patient inclusion criteria were used: i) Intermediate stage HCC (BCLC stage B) according to the American Association for the Study of Liver Diseases guidelines (15,27); ii) age $>18$ years; iii) no previous treatment with TACE for HCC; iv) World Health Organization performance status (PS) 0 , and v) complete follow-up from the initial treatment for HCC until death or the study censor date (November 30, 2018). The exclusion criteria were as follows: i) history of a malignant tumor other than $\mathrm{HCC}$ in the 5 years preceding this study; ii) participation in any drug trial; iii) BCLC stage $0, \mathrm{~A}, \mathrm{C}$, or D; iv) PS $>1$; v) creatinine $>1.5 \mathrm{mg} / \mathrm{dl}$; vi) infiltrative $\mathrm{HCC}$; vii) presence of portal vein tumor thrombosis or extrahepatic metastasis, viii) uncontrollable ascites; ix) active esophageal varices, and $\mathrm{x})$ received liver transplantation.
Evaluation of liver function. Liver function was evaluated by Child-Pugh score, was scored with five clinical measures of liver disease, such as total bilirubin level, serum albumin level, prothrombin activity, ascites (none/mild/moderate-severe), hepatic encephalopathy (none/grade I, II/grade III, IV). For example, Child-Pugh class A is 5-6 points and least severe liver disease (28).

Diagnosis and distribution of HCC. HCC was diagnosed by a tumor biopsy or a combination of tests for serum tumor makers such as alpha-fetoprotein (AFP) and des- $\gamma$-carboxy prothrombin (DCP) and imaging procedures such as ultrasonography, computed tomography, magnetic resonance imaging, and/or angiography.

For evaluation of distribution of HCC, we used arteriobiliary segmentation of liver (Healey and Schroy classification), which is consisted of following 5 segments: Anterior, posterior, medial, lateral segments, and caudate lobe (29). Regardless of the number of nodules, it defined the number of the liver segments occupied with nodules.

Treatment for HCC. TACE was selected based on the evidence-based clinical practice guidelines for HCC of-BCLC staging and treatment strategy (15).

TACE procedure. The hepatologist who performed the TACE procedures had more than 10 years of experience in interventional therapy at the start of this study. TACE was performed for the celiac artery and the common hepatic artery, which were catheterized with a 3 or $4 \mathrm{Fr}$ catheter, and digital subtraction angiography was performed with a nonionic iodine contrast agent. After evaluation of the tumor-containing segment using imaging techniques including cone-beam computed tomography, a 1.7 or $1.9 \mathrm{Fr}$ microcatheter (Piolax Inc.) was inserted into the sub- or sub-sub-hepatic segment to locate the tumor using the adapted microwire (Piolax Inc.). The catheter was advanced toward the tumor-feeding artery. Then, in conventional TACE, epirubicin was manually emulsified with lipiodol (Guerbet Co., Ltd.) depending on the size and number of tumors, and was administered followed by embolization with absorbable gelatin sponge particles (Nippon Kayaku Co., Ltd.) (30). A total of $20-50 \mathrm{mg}$ of epirubicin or cisplatin was used. In the drug-eluting (DC) beads-TACE procedure, $30 \mathrm{mg}$ of epirubicin was dissolved in $2 \mathrm{ml}$ saline and loaded into the DC Beads (Eisai Co., Ltd.). After epirubicin loading, the DC Beads containing epirubicin were diluted with $18 \mathrm{ml}$ of a dilution solution (1:1 saline/contrast agent). The particle size of the DC Beads was 100-300 $\mu \mathrm{m}$. All loading procedures were performed according to the manufacturer's recommended protocol (31). After catheterization into the artery that flowed into the area where the tumor was located, the diluted DC beads were administered slowly into the artery. DC beads were administered until the disappearance of blood flow.

Follow-up schedule after treatment of HCC. The first follow-up visit was performed approximately 1 month after TACE to assess the therapeutic efficacy, and the patients were followed up every 3 months until death or the study censor time (November 30, 2018). Each follow-up consisted of a physical examination, serum AFP and DCP examinations, 
and at least one imaging examination (abdominal ultrasound, enhanced computed tomography, or magnetic resonance imaging). Modified response evaluation criteria in solid tumors (RECIST) was used as the standard response criterion (32); $\mathrm{CR}$ is the disappearance of any intratumoral arterial enhancement in all target lesions, partial response (PR) is at least a $30 \%$ decrease in the sum of diameters of viable (contrast enhancement in the arterial phase) target lesions, taking as reference the baseline sum of the diameters of target lesions, progression disease (PD) is an increase of at least $20 \%$ in the sum of the diameters of viable (enhancing) target lesions, taking as reference the smallest sum of the diameters of viable (enhancing) target lesions recorded since the treatment started, stable disease (SD) is any cases that do not qualify for either PR or progressive disease. When HCC recurred, additional treatment for HCC was selected based on the evidence-based clinical practice guidelines for HCC of the BCLC staging and treatment strategy (15).

Additional treatment for recurrence of HCC after initial $T A C E$. For the recurrence of HCC after initial TACE, further TACE was generally employed according to the evidence-based clinical practice guidelines for $\mathrm{HCC}$ of the BCLC staging and treatment strategy (15). However, for the 14 patients with intermediate stage $\mathrm{HCC}$ and Child-Pugh class A who refused further TACE, MKIs were selected for the treatment [sorafenib alone $(n=11)$, sorafenib and regorafenib $(n=1)$, and lenvatinib $(n=2)]$.

Safety evaluation. Adverse events (AEs) and serious adverse events (SAEs) were monitored and recorded. AEs were assessed during both the treatment and the follow-up periods. AEs were assessed according to the National Cancer Institute Common Terminology Criteria for Adverse Events (CTCAE) version 4.0. For this study, adverse events were defined as those classified as greater than grade 3 according to CTCAE.

Clinical outcomes. The primary endpoint of this study was the OS of the patients.

Decision-tree algorithm. A decision-tree algorithm was constructed to reveal profiles associated with the prognosis of treatment with TACE-HCC and CR after initial TACE according to the instructions provided with the $\mathrm{R}$ software package as previously described (6). Following variables were used for the decision-tree analysis for OS and CR: gender, age, cause of HCC, Child-Pugh score, mRECIST, maximum nodule diameter, number of nodules, up-to-seven criteria, number of liver segments with nodule, gross classification, AFP level, and DCP level.

Statistical analysis. All data are expressed as the number or median (range). All statistical analyses were carried out using a statistical analysis software (JMP Pro v.13; SAS Institute Inc.). OS was calculated by the Kaplan-Meier method and analyzed by the log-rank test and Bonferroni methods. Factors associated with OS and CR were evaluated using multivariate stepwise analysis and decision-tree analysis. A two-tailed $\mathrm{P}<0.05$ was considered to indicate a statistically significant difference.
Table I. Patient characteristics.

\begin{tabular}{|c|c|}
\hline Characteristics & $\begin{array}{c}\text { Median (range) } \\
\text { or n }(\%)\end{array}$ \\
\hline Number & 238 \\
\hline Therapy (C-TACE/DC Beads-TACE) & $218 / 20$ \\
\hline Age, years (range) & $74(48-88)$ \\
\hline Sex (female/male) & $80(33.6) / 158(66.4)$ \\
\hline Cause of HCC (HBV/HCV/Others) & $12(6) / 185(77) / 41(17)$ \\
\hline Child-Pugh score (A/B) & $163(68) / 75(32)$ \\
\hline Maximum nodule diameter, $\mathrm{mm}$ & $31(10-127)$ \\
\hline \multicolumn{2}{|l|}{ Number of nodules } \\
\hline 1 & $55(23)$ \\
\hline 2 & $29(13)$ \\
\hline 3 & $21(9)$ \\
\hline 4 & $46(19)$ \\
\hline 5 & $36(15)$ \\
\hline$>6$ & $51(21)$ \\
\hline Up-to-seven criteria (within/out) & $135(56) / 103(42)$ \\
\hline \multicolumn{2}{|l|}{$\begin{array}{l}\text { Number of liver segments with } \\
\text { tumor }\end{array}$} \\
\hline$(<3 / \geq 3)$ & $120(50.5) / 118(49.5)$ \\
\hline $\begin{array}{l}\text { Gross classification } \\
\text { (simple nodular/other than } \\
\text { simple nodular) }\end{array}$ & $142(59) / 96(41)$ \\
\hline AFP, ng/ml & $36.2(1.8-62,546)$ \\
\hline $\mathrm{DCP}, \mathrm{mAU} / \mathrm{ml}$ & $83(9-75,000)$ \\
\hline \multicolumn{2}{|c|}{$\begin{array}{l}\text { Data are expressed as median (range) or number (\%). TACE, trans- } \\
\text { catheter arterial chemoembolization; C-TACE, conventional TACE; } \\
\text { DC Beads-TACE, Drug-eluting Bead TACE; HCC, hepatocellular } \\
\text { carcinoma; HBV, hepatitis B virus; HCV, hepatis C virus; AFP, } \\
\alpha \text {-fetoprotein; DCP, des- } \gamma \text {-carboxy prothrombin. }\end{array}$} \\
\hline
\end{tabular}

\section{Results}

Patient characteristics. The patients' median age was 74 years, and $33.6 \%$ of the patients $(80 / 238)$ were female (Table I). The etiology of $\mathrm{HCC}$ was hepatitis $\mathrm{C}$ virus in $77 \%$ of patients $(185 / 238)$, and $68 \%$ of patients (163/238) showed Child-Pugh class A. The median tumor size was $31 \mathrm{~mm}$, multiple nodules were seen in $76 \%$ of patients $(183 / 238)$, and $56 \%$ of patients $(135 / 238)$ were within the up-to-seven criteria which means within 7 being the sum of the maximum size and number of tumors for any given HCC (33) (Table I). Fifty-one percent $(120 / 238)$ of patients had $<3$ liver segments with nodule. In gross classification, in $59 \%$ of patients $(142 / 238)$ had simple nodular type (Table I).

Evaluation with mRECIST after initial TACE. An overall CR was observed in $27 \%$ of patients (65/238), PR in $32 \%$ of patients (77/238), stable disease (SD) in $11 \%$ of patients (28/238), and progressive disease (PD) in $30 \%$ of patients $(68 / 238)$. The objective response was $59 \%$, and the disease control rate was $70 \%$. 
A

Model building

$\mathrm{n}=\mathbf{2 3 8}$



B

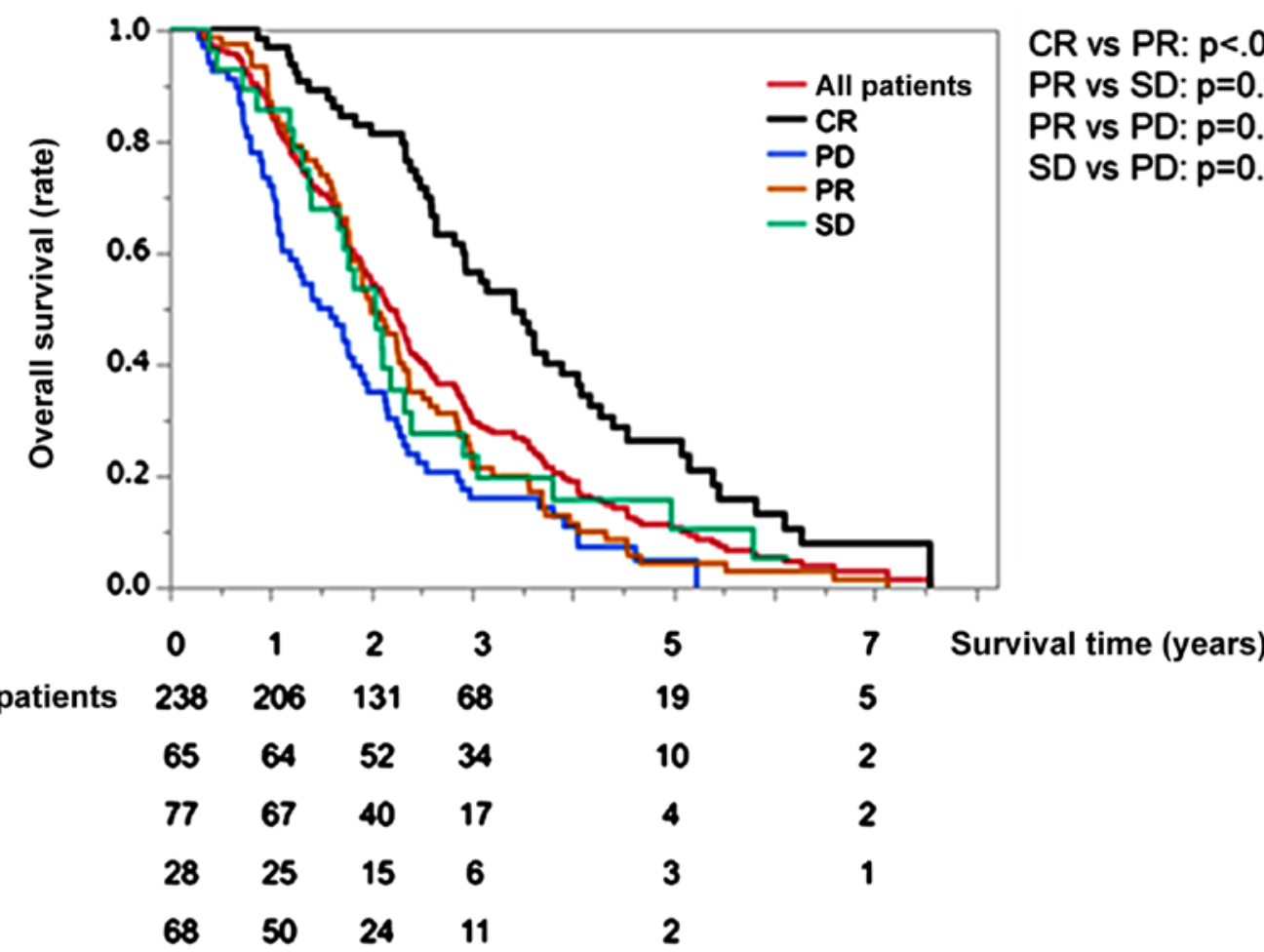

Figure 1. Decision-tree algorithm for survival and OS time in patients with intermediate stage HCC. (A) Subjects were classified according to the indicated cut-off values of the variables. The pie graphs indicate the percentage of living (white)/deceased (black) patients in each group. (B) Kaplan-Meier curves for OS in all patients, and according to mRECIST for initial TACE treatment. The MST was 26.6 months in all patients. In the present study, MST was defined as the length of time after which 50\% of the patients had died from the first TACE for HCC. OS, overall survival; HCC, hepatocellular carcinoma; mRECIST, modified response evaluation criteria in solid tumors; CR, complete response; PR, partial response; SD, stable disease; PD, progressive disease; MST, median survival time; TACE, transcatheter arterial chemoembolization.

$O S$ rate and decision-tree analysis for $O S$ in patients with intermediate stage HCC treated with initial TACE. The OS rates were 86,28 , and $8 \%$ at 1,3 , and 5 years, respectively. The median survival time (MST) was 26.6 months. At the study censor time, $10 \%(24 / 238)$ of included subjects were alive. Decision-tree analysis for OS demonstrated that mRECIST was selected as the variable for the initial split, and $21 \%$ of patients with CR were alive (Fig. 1A). In patients with PR, SD, and PD, gross classification was selected as the second split, and $10 \%$ of patients with simple nodular type were alive (Fig. 1A).

OS rate according to $m R E C I S T$. In the CR group, the $\mathrm{OS}$ rates were 98,52 , and $15 \%$ at 1,3 , and 5 years, respectively (Fig. 1B). Meanwhile, the rates were 87, 22, and 5\% in the PR 
group, 89,24 , and $10 \%$ in the SD group, and 73,16 , and $2 \%$ in the PD group (Fig. 1B). According to bonferroni methods, the OS rate in the CR group was significantly higher than that in the PR, SD, and PD groups (all $\mathrm{P}<.0001$ ). Also, the OS rate in the PR group was significantly higher than that in the PD group $(\mathrm{P}=0.007)$. On the other hands, there was no significant difference in the OS rate between the PR group and the SD groups $(\mathrm{P}=0.7686)$, and between the $\mathrm{SD}$ group and the $\mathrm{PD}$ group $(\mathrm{P}=0.1165$; Fig. $1 \mathrm{~B})$.

Decision-tree analysis for $C R$. In this study, CR rate was $27 \%(65 / 238)$ at the study censor time. To determine the profile for CR, a decision-tree analysis was performed. A decision-tree analysis identified the optimal number of liver segments to distinguish between Non-CR and CR, which nodule localized was selected as the variable for the initial split, and the CR rate was $51 \%$ in patients with $<3$ liver segments with nodule (Fig. 2A). In patients with $<3$ liver segments with nodule, gross classification was selected as the second split, and the CR rate was $64 \%$ in patients with simple nodular type (Profile 1; Fig. 2A). In patients with disease other than simple nodular type, the up-to-seven criteria were selected as the third split, and the CR rate was $35 \%$ for patients within the up-to-seven criteria (Profile 2; Fig. 2A). Meanwhile, the CR rate was 6 and 3\% in Profiles 3 and 4, respectively (Fig. 2A).

Logistic regression analysis for $C R$. Fewer than 3 liver segments with nodule, simple nodular type, and within the up-to-seven criteria were selected as the variables in a logistic regression analysis by a stepwise procedure. In the logistic regression analysis, all 3 variables were identified as independent factors for CR (Table II).

Difference in the $O S$ among each profile based on decision-tree analysis for $C R$. In Profile 1 , the OS rates were 93, 45, and $13 \%$ at 1,3 , and 5 years, respectively (Fig. 2B). In Profile 2, the OS rates were 96,35 , and $3 \%$ at 1,3 , and 5 years, respectively. Meanwhile, the OS rates were 86,26 , and $6 \%$ in Profile 3 and was 79, 16, and 3\% in Profile 4 at 1, 3, and 5 years, respectively (Fig. 2B). The OS rate in the Profile 1 was significantly higher than that in the Profile 2, Profile 3 , and Profile 4 groups $(\mathrm{P}=0.006,<.0001$, and $<.0001$, respectively). On the other hands, there was no significant difference in the OS rate between the Profile 2 and the Profile 3 or Profile 4 $(\mathrm{P}=0.057$ and $\mathrm{P}=0.073$, respectively), and between the Profile 3 and the Profile 4 ( $\mathrm{P}=0.837$; Fig. $2 \mathrm{~B})$. MST was 36.3 months in Profile 1, 28.2 months in Profile 2, 17.2 months in Profile 3 , and 21.6 months in Profile 4 (Fig. 2B). The MST of Profile 1 and 2 was longer than that of all patients (26.6 months), and these 2 profiles were categorized as the Better Profile. Meanwhile, the MST of Profiles 3 and 4 was shorter than that of all patients, and these 2 profiles were categorized as the Worse Profile. The MST was 35.7 months in the Better Profile and 21.6 months in the Worse Profile. There was a significant difference in OS between the Better Profile and the Worse Profile (Fig. 2C).

Patients' characteristics for the worse profile with child-pugh class $A$. To investigate the impact of MKIs for patients with
HCC recurrence after TACE, we analyzed patients with Child-Pugh class A in the Worse Profile. The median age was 73 years, and $27 \%$ of patients (24/87) were female (Table III). The etiology for HCC was hepatitis C virus in $79 \%$ of these patients (69/87). The median tumor size was $24 \mathrm{~mm}, 81 \%$ of patients $(71 / 87)$ had multiple nodules, and $31 \%$ of patients (27/87) were within the up-to-seven criteria. In gross classification, simple nodular type was seen in $59 \%$ of patients (51/87). For HCC recurrence after TACE, MKIs were selected for $16.1 \%$ of patients (14/87). Meanwhile, further TACE was selected for $83.9 \%$ of patients (73/87) (Table III).

Decision-tree analysis for $O S$ in the worse profile with child-pugh class A. The OS rate was $9 \%$ at the study censor time. MKIs were selected as the variable for the initial split. Although the OS rate was $4 \%$ in patients with further TACE, the OS rate was $35 \%$ in patients treated with MKIs (Fig. 3A).

Difference in OS between patients treated with MKIs and further TACE in the worse profile with child-pugh class A. In patients who were switched to MKIs, the OS was 100,57, and $14 \%$ at 1,3 , and 5 years, respectively (Fig. 3B). Meanwhile, the OS was 83,19 , and $5 \%$, respectively, in patients treated with further TACE. The OS rate in patients switched to MKIs was significantly higher than that in patients who underwent further TACE (Fig. 3B). The MST was 44.9 months in patients switched to MKIs and 21.9 months in patients who underwent further TACE.

Logistic regression analysis for the prognosis of patients with the worse profile and child-pugh class A. Switching to MKIs and gross classification were selected as the variables in a logistic regression analysis by a stepwise procedure. In the logistic regression analysis, both switching to MKIs and simple nodular type were identified as independent factors for prognosis (Table IV).

$A E$ and $S A E$. Among all patients, 2 patients (0.4\%) experienced SAEs that were assessed as greater than grade 3 AEs according to CTCAE. The complication was hepatic failure in $2(0.4 \%)$ patients after the initial TACE. However, these patients recovered completely with no aftereffects.

\section{Discussion}

In the present study, we demonstrated that CR by initial TACE was the most important variable for OS in patients with intermediate stage HCC. We found that profiles associated with a high CR rate were '<3 liver segments with nodule,' 'simple nodular type,' and 'within the up-to-seven criteria.', which are considered as suitable TACE criteria. Moreover, we showed that, in patients with ineligible for suitable TACE criteria, switching to MKIs may improve the prognosis to a greater degree than further TACE in cases of HCC recurrence after initial TACE.

TACE is a recommended therapy for intermediate stage HCC in the clinical guidelines for HCC worldwide $(15,34)$. In this study, we demonstrated that the MST was 26.6 months in patients with intermediate stage $\mathrm{HCC}$ who underwent initial TACE. Since the MST of intermediate stage HCC has been 
A

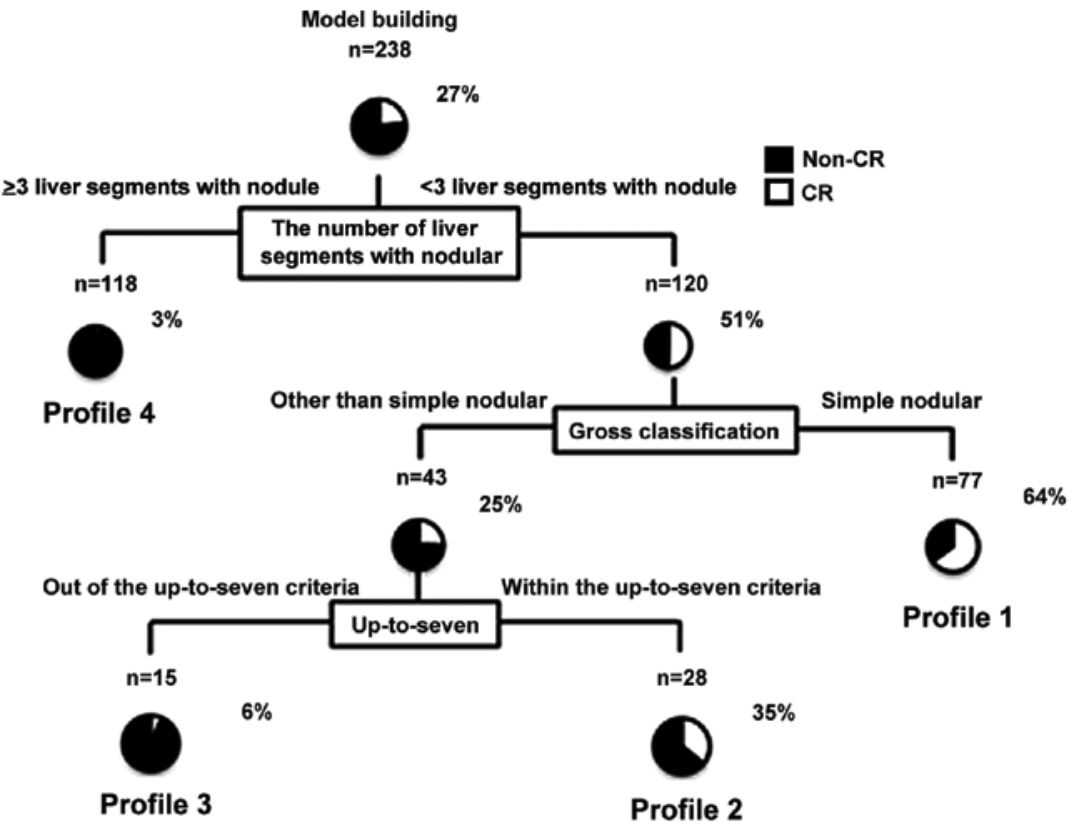

B

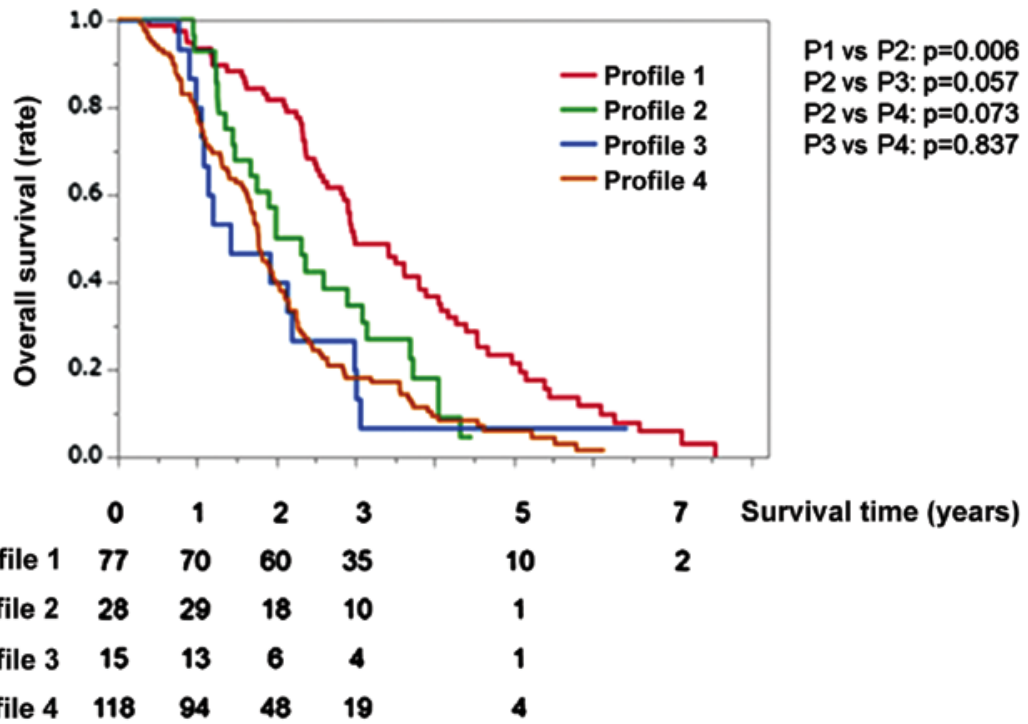

C

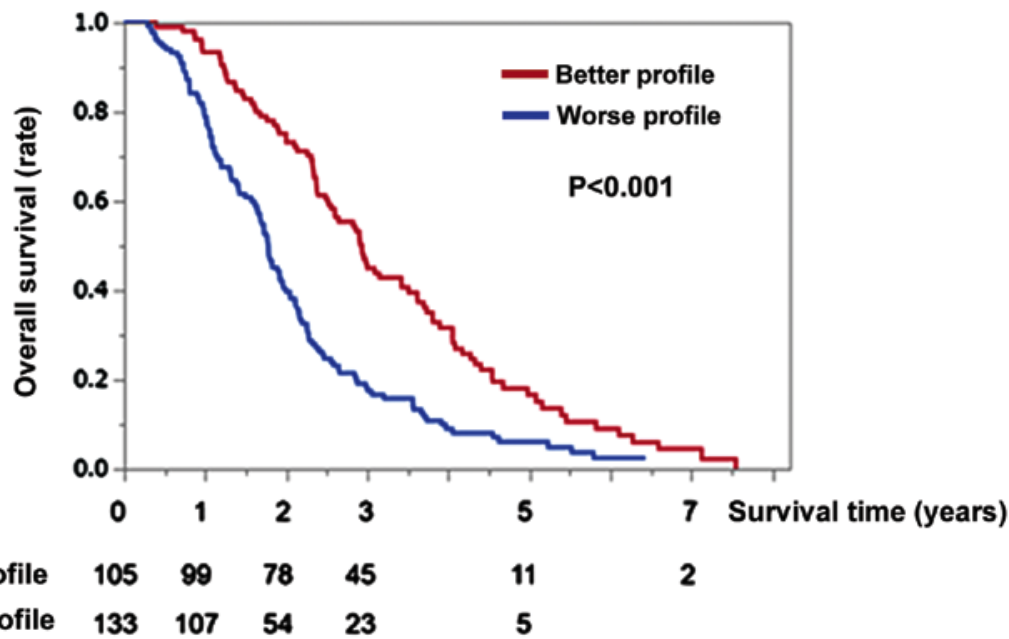

Figure 2. Profiles associated with CR after initial transcatheter arterial chemoembolization and OS time according to the profiles. (A) Decision-tree algorithm for CR. The pie graphs indicate the percentage of CR (white)/non-CR (black) patients in each group. (B) Kaplan-Meier curves for OS. (C) Kaplan-Meier curves for OS according to the Better and Worse Profiles. The Better Profile included $<3$ liver segments with nodule, simple nodular type and within the up-to-seven criteria. The Worse Profile includes $\geq 3$ liver segments with nodule, other than simple nodular type, and above the up-to-seven criteria. CR, complete response; OS, overall survival. 
Table II. Multivariate analysis for complete response.

\begin{tabular}{lrlr}
\hline Factors & $\begin{array}{c}\text { Odds } \\
\text { ratio }\end{array}$ & $95 \%$ CI & P-value \\
\hline$<3$ liver segments with nodule & 21.73 & $8.06-76.92$ & $<0.0001$ \\
Simple nodular type & 4.18 & $1.89-9.79$ & 0.0003 \\
Within the up-to-seven criteria & 3.52 & $1.39-9.76$ & 0.0072 \\
\hline
\end{tabular}

Table III. Patient characteristics for the Worse Profile with Child-Pugh A.

\begin{tabular}{lc}
\hline Characteristics & $\begin{array}{c}\text { All patients, mean } \\
\text { (range) or n }(\%)\end{array}$ \\
\hline Number & 87 \\
Age, years & $73(52-88)$ \\
Sex (female/male) & $24(27) / 63(73)$ \\
Cause of HCC (HBV/HCV/Others) & $6(7) / 69(79) / 12(14)$ \\
Maximum nodule diameter, mm & $24(10-127)$ \\
Number of nodules & \\
1 & $4(5)$ \\
2 & $4(5)$ \\
3 & $8(10)$ \\
4 & $17(19)$ \\
5 & $22(25)$ \\
$>6$ & $32(36)$ \\
Up-to-seven criteria (within/out) & $27(31) / 60(69)$ \\
Gross classification & $45(59) / 42(51)$ \\
(simple nodular/other than simple & \\
nodular) & \\
AFP, ng/ml & \\
DCP, mAU/ml & $36.2(1.8-62,546)$ \\
Therapy following TACE (MKIs/further & $14(16) / 73(84)$ \\
TACE) & \\
\hline & \\
\hline
\end{tabular}

Data are expressed as median (range) or number $(\%)$. HCC, hepatocellular carcinoma; $\mathrm{HBV}$, hepatitis B virus; $\mathrm{HCV}$, hepatis $\mathrm{C}$ virus; AFP, $\alpha$-fetoprotein; DCP, des- $\gamma$-carboxy prothrombin; TACE, transcatheter arterial chemoembolization; MKIs; multi-kinase inhibitors.

reported to be about 20 months (15), the prognosis of the patients in our study seems to be better than that in previous reports $(15,27,35)$. HCC treatment is reported to affect the prognosis of patients with HCC (11). It is important to identify the best candidates for TACE to prolong the OS of patients with intermediate stage HCC.

A combination of Child-Pugh class and the up-to-seven criteria has been used as the indication for TACE (36). TACE is recommended for $\mathrm{HCC}$ within the up-to-seven criteria (BCLC-B1) with an MST of 41.0 months (36). However, TACE was also recommended for HCC above the up-to-seven criteria (BCLC-B2 and BCLC-B3), and the MST of BCLC-B2 and BCLC-B3 has been reported to be 22.1 and 14.1 months, respectively (36). In our study, the CR for initial TACE was
Table IV. Multivariate analysis for prognosis in patients with the Worse Profile with Child-Pugh class A.

\begin{tabular}{lccr}
\hline Factors & Odds ratio & $95 \%$ CI & P-value \\
\hline Switching to MKIs & 0.26 & $0.11-0.56$ & $<0.001$ \\
Gross classification & 0.42 & $0.23-0.74$ & 0.002 \\
(simple nodular) & & & \\
\hline
\end{tabular}

MKIs, multi-kinase inhibitors.

selected as the first split factor for OS. The CR was associated with the following two profiles: Profile 1) ' $<3$ liver segments with nodule' and 'simple nodular type' and Profile 2) '<3 liver segments with nodule,' 'other than simple nodule,' and 'within the up-to-seven criteria.' The MST was 35.7 months in patients with Profiles 1 and 2 (Better Profile). Thus, besides being within the up-to-seven criteria, patients with these 2 profiles are thought to be suitable for treatment with TACE. Nevertheless, the up-to-seven criteria has been reported as a prognostic factor for TACE therapy $(36,37)$, patients with the Profile 1 were divided into 'within the up-to-seven criteria' (Profile 1a; $n=67$ ) or 'out of the up-to-seven criteria' (Profile $1 b ; n=10)$. The CR rate was 67 and $50 \%$ in the Profile $1 \mathrm{a}$ and $1 \mathrm{~b}$ groups, respectively (Fig. S1). There was no significant difference in the survival time between Profile $1 \mathrm{a}$ and $1 \mathrm{~b}(\mathrm{P}=0.691$; Fig. S2). These findings further support our hypothesis that gross classification was more important than up-to-seven criteria for OS in intermediate stage HCC patients treated with TACE Based on these findings, we demonstrated tumor development and tumor properties such as $<3$ liver segments with nodule and simple nodular type to be more important prognostic factors than the up-to-seven criteria for TACE in this study.

Previous studies demonstrated that sorafenib improved OS in TACE-refractory patients with intermediate stage HCC $(23,24,38)$, and the MST was prolonged by about 12 months with conversion to sorafenib compared to the MST with further TACE $(23,38)$. However, the effects of MKIs on OS remain unclear for patients with intermediate stage HCC who undergo treatments other than refractory TACE. In our study, we demonstrated that switching to MKIs improved the OS rates compared to further TACE in patients with HCC recurrence who did not meet our TACE criteria, in other words, those with 1) ' $\geq 3$ liver segments with noduler', 2) 'other than simple nodule,' and 3) 'out of the up-to-seven criteria'.

In this study, it was difficult to achieve CR with further TACE in such patients. Moreover, further TACE is known to worsen liver function $(18,39)$. Furthermore, TACE could induce a significant neoangiogenetic reaction by an increase of vascular endothelial growth factor level, which is a prognostic parameter of patients with HCC (40). Meanwhile, MKIs block vascular endothelial growth factor receptors, leading to suppression of tumor angiogenesis (41). Moreover, tumor growth related molecules such as Raf-1, c-kit, and platelet-derived growth factors are known prognostic factors for patients with HCC (42-44). Since MKIs inhibit Raf-1, c-kit, and platelet-derived growth factor signaling cascade (45), 
A

$$
\begin{gathered}
\text { Model building } \\
\mathrm{n}=87
\end{gathered}
$$

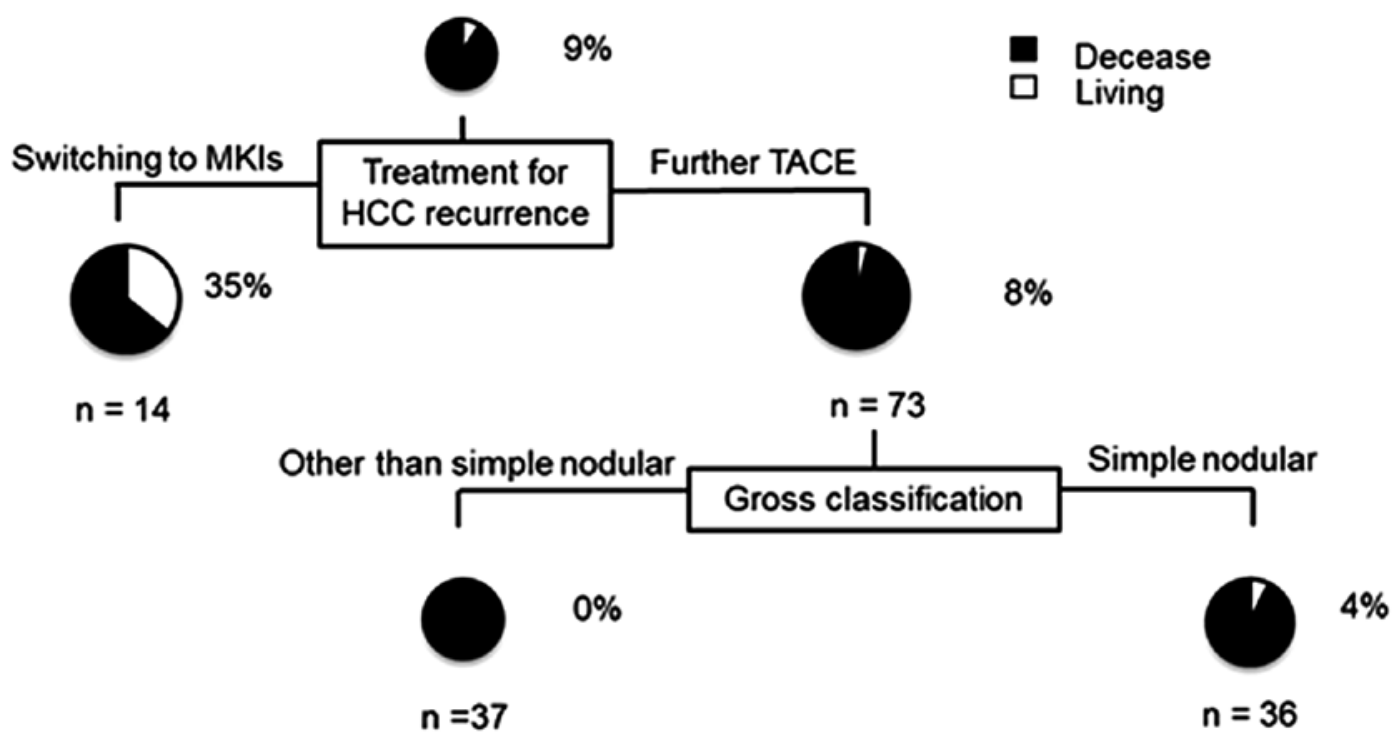

B

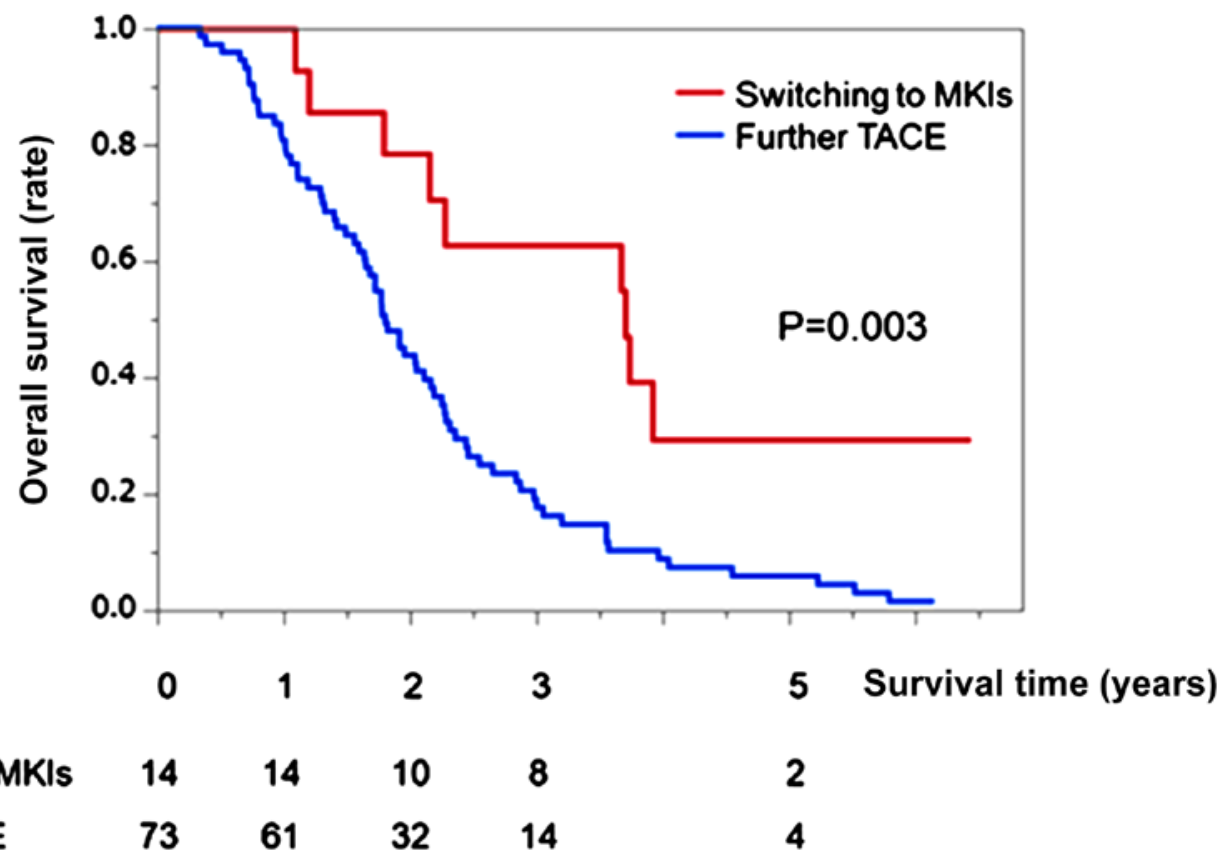

Figure 3. Profiles associated with survival in patients with the Worse Profile with Child-Pugh class A. (A) Decision-tree algorithm for survival in the Worse Profile with Child-Pugh class A. The pie graphs indicate the percentage of living (white)/deceased (black) patients in each group. (B) Kaplan-Meier curves for OS according to switching to MKIs and undergoing further TACE. HCC, hepatocellular carcinoma; OS, overall survival; MKIs, multi-kinase inhibitors; TACE, transcatheter arterial chemoembolization.

MKIs may improve the prognosis of patients with HCC through suppression of tumor angiogenesis and tumor growth. Taken together, switching to MKIs may improve the survival rate compared to further TACE in patients with HCC recurrence who do not meet the suitable TACE criteria.

The present study has several limitations. First, the study design was retrospective. Second, selection bias exists for the classification of the MKI and further TACE groups. Third, we did not evaluate the impact of progression-free survival on OS.
Fourth, we do not have a weighted test program. However, a weighted test should be used for survival plots where crossover between the groups is observed. Thus, a randomized controlled prospective validation study, which incorporates progression-free survival, is required in large number of patients with intermediate stage HCC to determine the indications for suitable TACE and the optimal timing to switch to MKIs.

In conclusion, the indications for suitable TACE in patients with intermediate stage $\mathrm{HCC}$ may be $<3$ liver segments with 
nodule,' 'simple nodular type,' and 'within the up-to-seven criteria.' Moreover, in patients with HCC who do not meet the criteria for suitable TACE, switching to MKIs may be associated with a better prognosis than further TACE in patients with HCC recurrence after TACE.

\section{Acknowledgements}

The authors would like to thank Drs. Ryoko Kuromatsu, Masahito Nakano, Shusuke Okamura and Yu Noda (all affilited with Division of Gastroenterology, Department of Medicine, Kurume University School of Medicine) for clinical management of patients.

\section{Funding}

No funding was received.

\section{Availability of data and materials}

The datasets used and/or analyzed during the present study are available from the corresponding author on reasonable request.

\section{Authors' contributions}

SS and MT participated in the study conception and design, acquisition of data, interpretation of data and drafting of the manuscript. HI, TN and TS participated in the acquisition of data and drafting of the manuscript. TK participated in the analysis and interpretation of data and drafting of the manuscript. HK and TT participated in the study conception and design and the critical revision. All authors agree to be accountable for all aspects of the work. All authors read and approved the final manuscript.

\section{Ethics approval and consent to participate}

The present study was approved by the ethical committee of Kurume University School of Medicine (approval no. 17205), and an opt-out approach was used to obtain informed consent from the patients.

\section{Patient consent for publication}

Not applicable.

\section{Competing interests}

TK received honoraria (lecture fees) from Mitsubishi Tanabe Pharma Corporation, MSD K.K., and Otsuka Pharmaceutical Co., Ltd. The other authors disclose no conflicts of interest.

\section{References}

1. Ferlay J, Soerjomataram I, Dikshit R, Eser S, Mathers C Rebelo M, Parkin DM, Forman D and Bray F: Cancer incidence and mortality worldwide: Sources, methods and major patterns in GLOBOCAN 2012. Int J Cancer 136: E359-E386, 2015.

2. Bruix J and Llovet JM: Prognostic prediction and treatment strategy in hepatocellular carcinoma. Hepatology 35: 519-524, 2002.
3. Belghiti $J$ and Fuks D: Liver resection and transplantation in hepatocellular carcinoma. Liver Cancer 1: 71-82, 2012.

4. Lee Cheah Y and P KHC: Liver transplantation for hepatocellular carcinoma: An appraisal of current controversies. Liver Cancer 1: 183-189, 2012.

5. Lin SM: Local ablation for hepatocellular carcinoma in Taiwan. Liver Cancer 2: 73-83, 2013.

6. Yamada S, Kawaguchi A, Kawaguchi T, Fukushima N, Kuromatsu R, Sumie S, Takata A, Nakano M, Satani M, Tonan $\mathrm{T}$, et al: Serum albumin level is a notable profiling factor for non-B, non- $\mathrm{C}$ hepatitis virus-related hepatocellular carcinoma: A data-mining analysis. Hepatol Res 44: 837-845, 2014.

7. Tateishi R, Uchino K, Fujiwara N, Takehara T, Okanoue $T$, Seike M, Yoshiji H, Yatsuhashi H, Shimizu M, Torimura T, et al: A nationwide survey on non-B, non-C hepatocellular carcinoma in Japan: 2011-2015 update. J Gastroenterol 54: 367-376, 2019.

8. Marasco G, Colecchia A, Colli A, Ravaioli F, Casazza G, Bacchi Reggiani ML, Cucchetti A, Cescon M and Festi D: Role of liver and spleen stiffness in predicting the recurrence of hepatocellular carcinoma after resection. J Hepatol 70: 440-448, 2019.

9. Nishikawa H, Kita R, Kimura T and Osaki Y: Transcatheter arterial embolic therapies for hepatocellular carcinoma: A literature review. Anticancer Res 34: 6877-6886, 2014.

10. Bruix J, Sala M and Llovet JM: Chemoembolization for hepatocellular carcinoma. Gastroenterology 127 (5 Suppl 1): S179-S188, 2004.

11. Takayasu K, Arii S, Ikai I, Omata M, Okita K, Ichida T, Matsuyama Y, Nakanuma Y, Kojiro M, Makuuchi M, et al: Prospective cohort study of transarterial chemoembolization for unresectable hepatocellular carcinoma in 8510 patients. Gastroenterology 131: 461-469, 2006.

12. Ando E, Tanaka M, Yamashita F, Kuromatsu R, Yutani S, Fukumori K, Sumie S, Yano Y, Okuda K and Sata M: Hepatic arterial infusion chemotherapy for advanced hepatocellular carcinoma with portal vein tumor thrombosis: Analysis of 48 cases. Cancer 95: 588-595, 2002.

13. Takeda H, Nishikawa H, Osaki Y, Tsuchiya K, Joko K, Ogawa C, Taniguchi H, Orito E, Uchida Y and Izumi N; Japanese Red Cross Liver Study Group: Clinical features associated with radiological response to sorafenib in unresectable hepatocellular carcinoma: A large multicenter study in Japan. Liver Int 35: 1581-1589, 2015.

14. Nakano $M$, Tanaka M, Kuromatsu R, Nagamatsu $H$, Sakata K, Matsugaki S, Kajiwara M, Fukuizumi K, Tajiri N, Matsukuma N, et al: Efficacy, safety, and survival factors for sorafenib treatment in Japanese patients with advanced hepatocellular carcinoma. Oncology 84: 108-114, 2013.

15. Forner A, Reig M and Bruix J: Hepatocellular carcinoma. Lancet 391: 1301-1314, 2018.

16. Uraki J, Yamakado K, Nakatsuka A and Takeda K: Transcatheter hepatic arterial chemoembolization for hepatocellular carcinoma invading the portal veins: Therapeutic effects and prognostic factors. Eur J Radiol 51: 12-18, 2004.

17. Piscaglia F and Ogasawara S: Patient selection for transarterial chemoembolization in hepatocellular carcinoma: Importance of benefit/risk assessment. Liver Cancer 7: 104-119, 2018.

18. Hiraoka A, Kumada T, Kudo M, Hirooka M, Koizumi Y, Hiasa Y, Tajiri K, Toyoda H, Tada T, Ochi H, et al: Hepatic function during repeated TACE procedures and prognosis after introducing sorafenib in patients with unresectable hepatocellular carcinoma: Multicenter analysis. Dig Dis 35: 602-610, 2017.

19. Wang W, Zhao Y, Bai W and Han G: Response assessment for HCC patients treated with repeated TACE: The optimal time-point is still an open issue. J Hepatol 63: 1530-1531, 2015.

20. Cheng AL, Kang YK, Chen Z, Tsao CJ, Qin S, Kim JS, Luo R, Feng J, Ye S, Yang TS, et al: Efficacy and safety of sorafenib in patients in the Asia-Pacific region with advanced hepatocellular carcinoma: A phase III randomised, double-blind, placebo-controlled trial. Lancet Oncol 10: 25-34, 2009.

21. Llovet JM, Ricci S, Mazzaferro V, Hilgard P, Gane E, Blanc JF, de Oliveira AC, Santoro A, Raoul JL, Forner A, et al: Sorafenib in advanced hepatocellular carcinoma. NEngl J Med 359: 378-390, 2008.

22. Kudo M, Finn RS, Qin S, Han KH, Ikeda K, Piscaglia F, Baron A, Park JW, Han G, Jassem J, et al: Lenvatinib versus sorafenib in first-line treatment of patients with unresectable hepatocellular carcinoma: A randomised phase 3 non-inferiority trial. Lancet 391: 1163-1173, 2018.

23. Ikeda M, Mitsunaga S, Shimizu S, Ohno I, Takahashi H, Okuyama H, Kuwahara A, Kondo S, Morizane C, Ueno H, et al: Efficacy of sorafenib in patients with hepatocellular carcinoma refractory to transcatheter arterial chemoembolization. J Gastroenterol 49: 932-940, 2014. 
24. Ogasawara S, Chiba T, Ooka Y, Kanogawa N, Motoyama T, Suzuki E, Tawada A, Kanai F, Yoshikawa M and Yokosuka O: Efficacy of sorafenib in intermediate-stage hepatocellular carcinoma patients refractory to transarterial chemoembolization. Oncology 87: 330-341, 2014.

25. de Jesus VHF and Dettino ALA: Update on hepatocellular carcinoma from the 2018 gastrointestinal cancer symposium (ASCO GI). J Hepatocell Carcinoma 5: 87-90, 2018.

26. Kudo M: Proposal of primary endpoints for TACE combination trials with systemic therapy: Lessons learned from 5 negative trials and the positive TACTICS trial. Liver Cancer 7: 225-234, 2018.

27. Heimbach JK, Kulik LM, Finn RS, Sirlin CB, Abecassis MM Roberts LR, Zhu AX, Murad MH and Marrero JA: AASLD guidelines for the treatment of hepatocellular carcinoma. Hepatology 67: 358-380, 2018.

28. Cholongitas E, Papatheodoridis GV, Vangeli M, Terreni N, Patch D and Burroughs AK: Systematic review: The model for end-stage liver disease-should it replace Child-Pugh's classification for assessing prognosis in cirrhosis? Aliment Pharmacol Ther 22: 1079-1089, 2005.

29. Healey JE Jr, Schroy PC and Sorensen RJ: The intrahepatic distribution of the hepatic artery in man. J Int Coll Surg 20 133-148, 1953

30. Horikawa M, Miyayama S, Irie T, Kaji T and Arai Y: Development of conventional transarterial chemoembolization for hepatocellular carcinomas in Japan: Historical, strategic, and technical review. AJR Am J Roentgenol 205: 764-773, 2015.

31. Lammer J, Malagari K, Vogl T, Pilleul F, Denys A, Watkinson A, Pitton M, Sergent G, Pfammatter T, Terraz S, et al: Prospective randomized study of doxorubicin-eluting-bead embolization in the treatment of hepatocellular carcinoma: Results of the PRECISION V study. Cardiovasc Intervent Radiol 33: 41-52, 2010.

32. Lencioni R and Llovet JM: Modified RECIST (mRECIST) assessment for hepatocellular carcinoma. Semin Liver Dis 30 : $52-60,2010$.

33. D'Amico F, Schwartz M, Vitale A, Tabrizian P, Roayaie S, Thung S, Guido M, del Rio Martin J, Schiano T and Cillo U: Predicting recurrence after liver transplantation in patients with hepatocellular carcinoma exceeding the up-to-seven criteria. Liver Transpl 15: 1278-1287, 2009.

34. Kokudo N, Hasegawa K, Akahane M, Igaki H, Izumi N, Ichida T, Uemoto S, Kaneko S, Kawasaki S, Ku Y, et al: Evidence-based clinical practice guidelines for hepatocellular carcinoma: The Japan society of hepatology 2013 update (3rd JSH-HCC Guidelines). Hepatol Res 45, 2015.

35. Han K and Kim JH: Transarterial chemoembolization in hepatocellular carcinoma treatment: Barcelona clinic liver cancer staging system. World J Gastroenterol 21: 10327-10335, 2015.
36. Bolondi L, Burroughs A, Dufour JF, Galle PR, Mazzaferro V, Piscaglia F, Raoul JL and Sangro B: Heterogeneity of patients with intermediate (BCLC B) hepatocellular carcinoma: Proposal for a subclassification to facilitate treatment decisions. Semin Liver Dis 32: 348-359, 2012.

37. YamakadoKandHirota S: Sub-classification of intermediate-stage (Barcelona Clinic Liver Cancer stage-B) hepatocellular carcinomas. World J Gastroenterol 21: 10604-10608, 2015.

38. Arizumi T, Ueshima K, Minami T, Kono M, Chishina $\mathrm{H}$, Takita M, Kitai S, Inoue $\mathrm{T}$, Yada $\mathrm{N}$, Hagiwara $\mathrm{S}$, et al: Effectiveness of sorafenib in patients with transcatheter arterial chemoembolization (TACE) refractory and intermediate-stage hepatocellular carcinoma. Liver Cancer 4: 253-262, 2015.

39. Ikeda M, Arai Y, Park SJ, Takeuchi Y, Anai H, Kim JK, Inaba Y, Aramaki T, Kwon SH, Yamamoto S, et al: Prospective study of transcatheter arterial chemoembolization for unresectable hepatocellular carcinoma: An Asian cooperative study between Japan and Korea. J Vasc Interv Radiol 24: 490-500, 2013

40. Sergio A, Cristofori C, Cardin R, Pivetta G, Ragazzi R, Baldan A, Girardi L, Cillo U, Burra P, Giacomin A and Farinati F: Transcatheter arterial chemoembolization (TACE) in hepatocellular carcinoma (HCC): The role of angiogenesis and invasiveness. Am J Gastroenterol 103: 914-921, 2008.

41. Reză̌kina AV and Komarova VD: An immunoenzyme method of detecting anti-DNA antibodies in lupus erythematosus. Lab Delo: 40-43, 1988 (In Russian).

42. Chen B, Liu J, Wang X, Shen Q, Li C and Dai C: Co-expression of PDGF-B and VEGFR-3 strongly correlates with poor prognosis in hepatocellular carcinoma patients after hepatectomy. Clin Res Hepatol Gastroenterol 42: 126-133, 2018.

43. Chen L, Shi Y, Jiang CY, Wei LX, Wang YL and Dai GH: Expression and prognostic role of pan-Ras, Raf-1, pMEK1 and pERK1/2 in patients with hepatocellular carcinoma. Eur J Surg Oncol 37: 513-520, 2011.

44. Yan W, Zhu Z, Pan F, Huang A and Dai GH: Overexpression of c-kit(CD117), relevant with microvessel density, is an independent survival prognostic factor for patients with HBV-related hepatocellular carcinoma. Onco Targets Ther 11: 1285-1292, 2018.

45. Weintraub JL and Salem R: Treatment of hepatocellular carcinoma combining sorafenib and transarterial locoregional therapy: State of the science. J Vasc Interv Radiol 24: 1123-1134, 2013.

This work is licensed under a Creative Commons Attribution-NonCommercial-NoDerivatives 4.0 International (CC BY-NC-ND 4.0) License. 